\title{
EKELAND'S VARIATIONAL PRINCIPLE FOR SET-VALUED FUNCTIONS*
}

\author{
C. G. LIU $\dagger$ AND K. F. NG ${ }^{\ddagger}$
}

\begin{abstract}
We establish several set-valued function versions of Ekeland's variational principle and hence provide some sufficient conditions ensuring the existence of error bounds for inequality systems defined by finitely many lower semicontinuous functions.
\end{abstract}

Key words. Ekeland's variational principle, partial order, error bound

AMS subject classifications. 58E $30,65 \mathrm{~K} 10,90 \mathrm{C} 31$

1. Introduction. The celebrated variational principle of Ekeland $[13,14]$ states that if $f$ is a lower semicontinuous (lsc) bounded below function on a complete metric space $(M, d)$ then for any $\gamma>0$ and any $x_{0}$ with $f\left(x_{0}\right)<+\infty$ there exists $\bar{x} \in M$ such that

$$
f(\bar{x}) \leq f\left(x_{0}\right)-\gamma d\left(x_{0}, \bar{x}\right)
$$

and

$$
f(\bar{x})<f(x)+\gamma d(x, \bar{x}), \quad \forall x \in M \backslash\{\bar{x}\} .
$$

This principle is an important tool with a lot of significant applications in many areas including nonlinear analysis and optimization theory. There are many papers (see $[6,8,11,35]$ for example) reporting different formulations and some have put forward extended versions applicable to vector-valued/set-valued functions (see [3, 4, $5,9,15,16,18,38])$. This paper is devoted to further extensions which are especially relevant for the study of error bound issue for the following inequality system:

$$
f_{i}(x) \leq 0, \quad i \in \overline{1, n},
$$

where each $f_{i}$ is a proper lsc function on a Banach space $X$. The system is said to have an error bound $\tau$ if there exists $\tau>0$ such that

$$
d(x, S) \leq \tau \sum_{i=1}^{n} f_{i}(x)_{+}, \quad \forall x \in X
$$

where $f_{i}(x)_{+}:=\max \left\{f_{i}(x), 0\right\}$, and

$$
S:=\left\{x \in X: f_{i}(x) \leq 0, i \in \overline{1, n}\right\} .
$$

Since the pioneering work of Hoffman [19], this notion (and its local version) have played an important role in many areas in mathematical programming and variational analysis (see the excellent surveys [23, 31] for results before 1997, and for more recent

\footnotetext{
*This research was supported by the National Natural Science Foundation of China (No. 10801137) and an Earmarked Grant (GRF) from the Research Grant Council of Hong Kong.

${ }^{\dagger}$ Department of Mathematics, Jinan University, Guangzhou, 510632, P.R.China. (tcgliu@jnu.edu.cn)

${ }^{\ddagger}$ Department of Mathematics (and IMS), The Chinese University of Hong Kong, Shatin, New Territory, Hong Kong. (kfng@math.cuhk.edu.hk)
} 
results see $[1,2,7,10,12,27,28,29,30,36]$ and references therein). The earlier results all are either under certain convexity assumption or restricted to the special case when $n=1$. The results obtained in Section 4 are for the general case that $n$ can be any positive integer, and provide sufficient conditions ensuring that the inequality system (1.3) has an error bound. They are established via set-valued versions of Ekeland's variational principle obtained in Section 3.

2. Notations and Preliminary Results. In general we use $(M, d)$ to denote a metric space while $X, Y$ and $Z$ usually denote normed spaces (or Banach spaces); $B(x, r)$ denotes the closed ball with center $x$ and radius $r>0$. For short $B_{Z}$ denotes the closed unit ball in $\mathrm{Z}$ and $S_{Z}:=\{z \in Z:\|z\|=1\}$ is the unit sphere. Let $\left\langle z^{*}, z\right\rangle:=z^{*}(z)$. Given any subset $K$ in $Z, S(K)=K \cap S_{Z}$. Let $c l(K), \operatorname{co}(K)$, $\overline{c o}(K)$, cone $K$ and $\overline{c o n e} K$ respectively denote the closure, convex hull, closed convex hull, (convex) conic hull, and closed (convex) conic hull of $K$. Let $d(\cdot, K)$ denote the distance function of $K$, i.e.,

$$
d(z, K):=\inf \{\|z-y\|: y \in K\} .
$$

We say that a vector space $Y$ is ordered by a convex cone $C \subset Y$, if $Y$ is equipped with a binary relation (quasiorder) $\leq_{C}$ for elements in $Y$ such that

$$
y_{1} \leq_{C} y_{2} \Longleftrightarrow y_{2}-y_{1} \in C
$$

( $\leq_{C}$ is a partial order if and only if $C$ is pointed, i.e., $C \cap(-C)=\{0\}$ ). For example, in multi-objective optimization problems, we often let $\mathbb{R}^{n}$ be ordered by $\mathbb{R}_{+}^{n}$, where $\mathbb{R}_{+}^{n}$ consists of all $n$-vectors $y=\left(y_{1}, \cdots, y_{n}\right) \in \mathbb{R}^{n}$ such that each $y_{i} \geq 0$. Here the norm for $\mathbb{R}^{n}$ can be the usual Euclidean norm $\|y\|=\left(\sum_{i=1}^{n}\left|y_{i}\right|^{2}\right)^{\frac{1}{2}}$ or the $l_{1}$-norm $\|y\|_{1}=\sum_{i=1}^{n}\left|y_{i}\right|$. One of the advantages of using the $l_{1}$-norm is that,

$$
\text { if } H:=\left\{y \in \mathbb{R}_{+}^{n}:\|y\|_{1}=1\right\} \text { then } d(0, H)=1
$$

(and $H$ is closed and convex). For convenience, we henceforth use the $l_{1}$-norm for $\mathbb{R}^{n}$.

As usual for a set-valued function $F: M \rightarrow 2^{Y}$, we use $\operatorname{dom} F$ and $\operatorname{graph} F$ to denote the domain and the graph of $F$ respectively, that is, $\operatorname{dom} F:=\{x \in M$ : $F(x) \neq \emptyset\}$, graph $F:=\{(x, y) \in M \times Y: y \in F(x)\}$. Moreover, if $Y$ is ordered by $C$, epi $F:=\{(x, y) \in M \times Y: y \in F(x)+C\}$ is the epigraph of $F$. Thus

$$
v \leq_{C} y, v \in F(x) \Longrightarrow(x, y) \in e p i F .
$$

For any two nontrivial closed convex cones $K_{1}, K_{2}$ in a normed space $Y$ we use $\measuredangle\left(K_{1}, K_{2}\right)$ to denote the quantity (cf. [24, equation (2.2)])

$$
\measuredangle\left(K_{1}, K_{2}\right):=\inf \left\{d\left(k, K_{2}\right): k \in S\left(K_{1}\right)\right\} .
$$

LEMma 2.1. Let $Y$ be a normed space ordered by a convex cone $C \subset Y$, and let $C_{0}$ be a convex cone such that $\{0\} \neq C_{0} \subset C$. Then the following implication is valid for all $y_{1} \in C_{0}$ and $y_{2} \in Y$ :

$$
y_{1} \leq_{C} y_{2} \Longrightarrow y_{2} \in C \text { and } \measuredangle\left(C_{0},-C\right)\left\|y_{1}\right\| \leq\left\|y_{2}\right\| .
$$

Proof. By (2.4), we have

$$
\measuredangle\left(C_{0},-C\right) \cdot\|y\| \leq d(y,-C), \quad \forall y \in C_{0} .
$$


Let $y_{1} \in C_{0}$ and $y_{2} \in Y$ with $y_{1} \leq_{C} y_{2}$. Then $y_{2} \in y_{1}+C \subset C$. Moreover since $y_{1}-y_{2} \in-C$, one has by $(2.6)$,

$$
\measuredangle\left(C_{0},-C\right) \cdot\left\|y_{1}\right\| \leq d\left(y_{1},-C\right) \leq\left\|y_{1}-\left(y_{1}-y_{2}\right)\right\|=\left\|y_{2}\right\| .
$$

This proves (2.5).

REMARK 2.1. It is well-known (see [32]) that $C$ is normal in $(Y,\|\cdot\|)$ (in the sense that any $\left\{y_{n}\right\}_{n \in \mathbb{N}}$ converges to zero whenever there are sequences $\left\{x_{n}\right\}_{n \in \mathbb{N}},\left\{z_{n}\right\}_{n \in \mathbb{N}}$ convergent to zero such that $x_{n} \leq_{C} y_{n} \leq_{C} z_{n}$ for each $n$ ) if and only if there is an equivalent norm $\|\cdot\|_{1}$ on $Y$ such that

$$
y_{1} \leq_{C} y_{2} \Longrightarrow\left\|y_{1}\right\|_{1} \leq\left\|y_{2}\right\|_{1}, \quad \forall y_{1}, y_{2} \in C .
$$

In view of (2.4) and Lemma 2.1, it follows immediate that $C$ is normal if and only if

$$
\measuredangle(C,-C)>0 .
$$

In particular, if (2.7) is satisfied with $\|\cdot\|$ in place of $\|\cdot\|_{1}$ (this condition is automatically satisfied if $Y$ is a Banach lattice with positive cone $C$; see [34, Definition II.1.2, II.5.1]). Then $\measuredangle\left(C_{0},-C\right)=1$ for any convex cone $C_{0}$ such that $\{0\} \neq C_{0} \subset C$. Indeed, if $y_{1} \in S\left(C_{0}\right)$ then $d\left(y_{1},-C\right)=\inf \left\{\left\|y_{1}+c\right\|: c \in C\right\}=\left\|y_{1}\right\|=1$.

Example 2.1. Let $Y$ be a Hilbert space. Fix $e \in S(Y)$ and let $C:=\{y \in Y$ : $\left.\langle e, y\rangle \geq \frac{1}{\sqrt{2}}\|y\|\right\}$. It can be verified that $\measuredangle\left(C_{0},-C\right)=1$ for any convex cone $C_{0}$ with $\{0\} \neq C_{0} \subset C$.

Let $A$ be a nonempty subset of a metric space $(M, d)$, and let $\preceq$ be a partial order defined on $A$. Recall that a point $\bar{a} \in A$ is called a minimal point of $A$ if there does not exist $a \in A \backslash\{\bar{a}\}$ such that $a \preceq \bar{a}$. The set of all the minimal points of $A$ is denoted by $\operatorname{Min}(A, \preceq)$. Recall that $A$ is said to have the domination property with respect to $\preceq$ if for each $x_{0} \in A$, there is $\bar{x} \in A$ such that

$$
\bar{x} \preceq x_{0} \quad \text { and } \quad \bar{x} \in \operatorname{Min}(A, \preceq) .
$$

The next result is due to Hamel and Tammer and would be convenient to be stated in the following form:

Lemma 2.2. Let $A$ be a nonempty subset of a metric space $(M, d)$, and let $\preceq$ be a partial order defined on $A$ such that any decreasing sequence $\left\{x_{n}\right\}_{n \in \mathbb{N}}$ in $A$ converges to some $\bar{a} \in A$ with

$$
\bar{a} \preceq x_{n} \quad \forall n \in \mathbb{N} .
$$

Then $A$ has the domination property with respect to $\preceq$.

Proof. This follows immediately from [18, Theorem 2.2].

3. Partial Orders Generated by Set-Valued Functions. Let $(M, d)$ be a complete metric space and let $Y$ be a Banach space ordered by a nontrivial closed convex cone $C$. Let $F: M \rightarrow 2^{Y}$ be a set-valued function.

Definition 3.1. Let $\gamma>0$ and let $H \subset C \backslash\{0\}$ be a closed convex set such that

$$
\measuredangle(\overline{c o n e} H,-C)>0 .
$$

We define relations $\preceq_{(F, \gamma, H)}$ and $\precsim_{(F, \gamma, H)}$ (or $\preceq, \precsim$ for short if no confusion can arise) on $\operatorname{dom} F$ by

$$
\begin{aligned}
& x_{1} \preceq_{(F, \gamma, H)} x_{2} \Longleftrightarrow \\
& \quad \forall y_{2} \in F\left(x_{2}\right), \exists y_{1} \in F\left(x_{1}\right), h \in H, \text { s.t. } \gamma d\left(x_{1}, x_{2}\right) h \leq_{C} y_{2}-y_{1},
\end{aligned}
$$


and

$$
x_{1} \precsim_{(F, \gamma, H)} x_{2} \Longleftrightarrow \forall \gamma^{\prime} \in(0, \gamma), x_{1} \preceq_{\left(F, \gamma^{\prime}, H\right)} x_{2}
$$

respectively, where $x_{1}, x_{2} \in \operatorname{dom} F$.

By (3.2), we have the following equivalences:

$$
\begin{aligned}
x_{1} \preceq_{(F, \gamma, H)} x_{2} & \Longleftrightarrow F\left(x_{2}\right) \subset F\left(x_{1}\right)+\gamma d\left(x_{1}, x_{2}\right) H+C \\
& \Longleftrightarrow \forall y_{2} \in F\left(x_{2}\right), \exists h \in H \text { s.t. }\left(x_{1}, y_{2}-\gamma d\left(x_{1}, x_{2}\right) h\right) \in \text { epi } F .
\end{aligned}
$$

Since $H \subset C$ and by (2.3), the following implications are also valid:

$$
\begin{aligned}
& x_{1} \preceq_{(F, \gamma, H)} x_{2} \Longrightarrow F\left(x_{2}\right) \subset F\left(x_{1}\right)+C, \\
& x_{1} \preceq_{(F, \gamma, H)} x_{2} \Longrightarrow\left(x_{1}, y_{2}\right) \in e p i F, \forall y_{2} \in F\left(x_{2}\right) .
\end{aligned}
$$

It is clear that for any $x_{1}, x_{2} \in \operatorname{dom} F$,

$$
x_{1} \preceq_{(F, \gamma, H)} x_{2} \Longrightarrow x_{1} \precsim_{(F, \gamma, H)} x_{2} .
$$

But generally, the inverse does not hold.

EXAMPLE 3.1. Let $Y=l_{2}$, the Hilbert space consisting of all square-summable sequences of real numbers. For each $n \in \mathbb{N}$, let $e_{n}$ denote the element in $l_{2}$ whose $n^{\text {th }}$ coordinate is 1 and other coordinates are zero. Let $C:=\left\{y \in l_{2}:\left\langle e_{1}, y\right\rangle \geq \frac{1}{\sqrt{2}}\|y\|\right\}$. Consider two distinct points $x_{1}, x_{2}$ in metric space $(M, d)$ with

$$
d\left(x_{1}, x_{2}\right)=1 .
$$

Let $H:=\left\{y \in C:\left\langle e_{1}, y\right\rangle=1\right\}$. Then $H$ is a closed convex subset of $C$ such that coneH $=C$ (so coneH is closed). By Example 2.1,

$$
\measuredangle(\overline{c o n e} H,-C)=1 \text {. }
$$

Let $F:\left\{x_{1}, x_{2}\right\} \rightarrow 2^{Y}$ be defined by

$$
\begin{aligned}
& F\left(x_{2}\right)=\{0\}, \\
& F\left(x_{1}\right)=\left\{\left(\frac{1}{n}-1\right)\left(e_{1}+e_{n}\right)\right\}_{n \geq 2} .
\end{aligned}
$$

We claim that

$$
x_{1} \precsim(F, 1, H) x_{2},
$$

but that

$$
x_{1} \npreceq_{(F, 1, H)} x_{2},
$$

First, since $e_{1}+e_{n} \in H$ for all $n \geq 2$, we have

$$
F\left(x_{2}\right) \subset F\left(x_{1}\right)+\left(1-\frac{1}{n}\right) H+C, \quad \forall n \geq 2 .
$$

Also, for any $\gamma^{\prime} \in(0,1)$, there exists $n^{\prime} \geq 2$ such that $\gamma^{\prime}<1-\frac{1}{n^{\prime}}$. Hence by (3.15) and the fact that $\left(1-\frac{1}{n^{\prime}}\right) H \subset \gamma^{\prime} H+C$, it follows from (3.9) that

$$
\begin{aligned}
F\left(x_{2}\right) & \subset F\left(x_{1}\right)+\gamma^{\prime} H+C \\
& =F\left(x_{1}\right)+\gamma^{\prime} d\left(x_{1}, x_{2}\right) H+C .
\end{aligned}
$$


Together with (3.3) and (3.4), we obtain (3.13).

Second, since $\left(\frac{1}{n}-1\right)\left(e_{1}+e_{n}\right) \notin-H-C$ for all $n \geq 2$, we have

$$
F\left(x_{1}\right) \cap(-H-C)=\emptyset,
$$

and then

$$
F\left(x_{2}\right)=\{0\} \nsubseteq F\left(x_{1}\right)+H+C .
$$

Together with (3.4), (3.14) holds.

Recall that a subset $D$ of $Y$ is said to be $C$-bounded (cf. [25, pp. 13-14]) if there exists some bounded set $D_{0} \subset Y$ such that $D \subset D_{0}+C$. For example, with $(Y, C)=\left(\mathbb{R}, \mathbb{R}_{+}\right)$, a subset $D$ of $\mathbb{R}$ is $\mathbb{R}_{+}$-bounded if and only if $D$ is a bounded below set of real numbers.

For the remainder of this section, the following assumptions will be considered:

(A1) $H \subset C \backslash\{0\}$ is a closed convex set (thus we have $\kappa:=d(0, H)>0$ ).

(A2) $\zeta:=\measuredangle(\overline{c o n e} H,-C)>0$.

(A3) $F(M)$ is $C$-bounded: there exists $\eta>0$ such that

$$
\forall y \in F(M), \exists u \in Y \text { s.t. }\|u\| \leq \eta \text { and } u \leq_{C} y .
$$

(A4) epi $F$ is closed in $M \times Y$.

EXAMPLE 3.2. In the ordered Banach space $(Y, C)=\left(\mathbb{R}^{n}, \mathbb{R}_{+}^{n}\right)$, let $H$ be defined as (2.2). Then (A1) is satisfied. Moveover, it is obvious that $\overline{\text { cone }} H=\mathbb{R}_{+}^{n}$ and

$$
\measuredangle\left(\overline{\text { cone }} H,-\mathbb{R}_{+}^{n}\right)=1 .
$$

So (A2) is also true.

For the inequality system (1.3), let $D:=\cap_{i=1}^{n} \operatorname{dom} f_{i}$ and let $F: X \rightarrow \mathbb{R}^{n}$ be a set-valued function defined as

$$
F(x):=\left\{\begin{array}{ll}
\left\{\left(f_{1}(x)_{+}, f_{2}(x)_{+}, \cdots, f_{n}(x)_{+}\right)\right\} & x \in D \\
\emptyset & x \notin D
\end{array} .\right.
$$

Then $F(X) \subset \mathbb{R}_{+}^{n}$ is $\mathbb{R}_{+}^{n}$-bounded. It is easy to verify that epi $F$ is closed. Thus (A3) and (A4) are satisfied.

The following proposition provides a sufficient condition ensuring that $\preceq$ and $\precsim$ are partial orders:

Proposition 3.2. Consider $\gamma>0$ and $F, H$ satisfying assumptions (A1)-(A3) with the associated constants $\kappa, \zeta, \eta>0$. Then, both the relations $\preceq_{(F, \gamma, H)}$ and $\precsim_{(F, \gamma, H)}$ defined in Definition 3.1 are partial orders on dom $F$.

Proof. We need only to show that the relation $\preceq$ is a partial order on $\operatorname{dom} F$ (as the corresponding result for $\precsim$ follows easily). It is easy to see that the relation $\preceq$ is reflexive, that is, $x \preceq x$ for all $x \in \operatorname{dom} F$. Let $x_{1}, x_{2}, x_{3}$ be distinct elements of $\operatorname{dom} F$ such that $x_{1} \preceq x_{2}$ and $x_{2} \preceq x_{3}$. Then for any $y_{3} \in F\left(x_{3}\right)$, there exist $y_{2} \in F\left(x_{2}\right), y_{1} \in F\left(x_{1}\right)$ and $h_{1}, h_{2} \in H$ such that $\gamma d\left(x_{1}, x_{2}\right) h_{1} \leq_{C} y_{2}-y_{1}$ and $\gamma d\left(x_{2}, x_{3}\right) h_{2} \leq_{C} y_{3}-y_{2}$. Let $h_{3}:=\frac{d\left(x_{1}, x_{2}\right)}{d\left(x_{1}, x_{2}\right)+d\left(x_{2}, x_{3}\right)} h_{1}+\frac{d\left(x_{2}, x_{3}\right)}{d\left(x_{1}, x_{2}\right)+d\left(x_{2}, x_{3}\right)} h_{2}$. Then $h_{3} \in H \subset C$ and

$$
\begin{aligned}
\gamma d\left(x_{1}, x_{3}\right) h_{3} & \leq_{C} \gamma\left[d\left(x_{1}, x_{2}\right)+d\left(x_{2}, x_{3}\right)\right] h_{3} \\
& \leq_{C} y_{3}-y_{1} .
\end{aligned}
$$


So $x_{1} \preceq x_{3}$. To prove the anti-symmetry of $\preceq$, suppose that $x \preceq x^{\prime}$ and $x^{\prime} \preceq x$. By what has just been proved, for any $z \in F(x)$, there exist $z^{\prime} \in F(x)$ and $h^{\prime} \in H$ such that

$$
2 \gamma d\left(x, x^{\prime}\right) h^{\prime} \leq_{C} z-z^{\prime} .
$$

Therefore, inductively, there exist sequences $\left\{z_{n}\right\}_{n \in \mathbb{N}} \subset F(x)$ and $\left\{h_{n}^{\prime}\right\}_{n \in \mathbb{N}} \subset H$ such that

$$
2 \gamma d\left(x, x^{\prime}\right) h_{n}^{\prime} \leq_{C} z_{n}-z_{n+1}, \quad \forall n \in \mathbb{N} .
$$

By assumption (A3), there exists a sequence $\left\{u_{n}\right\}_{n \in \mathbb{N}} \subset Z$ such that

$$
\left\|u_{n}\right\| \leq \eta, \quad \forall n \in \mathbb{N},
$$

and

$$
u_{n} \leq_{C} z_{n}, \quad \forall n \in \mathbb{N} .
$$

It follows from (3.24) and (3.26) that

$$
2 \gamma d\left(x, x^{\prime}\right) \sum_{i=1}^{n} h_{i}^{\prime} \leq_{C} z_{1}-z_{n+1} \leq_{C} z_{1}-u_{n+1}, \quad \forall n \in \mathbb{N} .
$$

Since $H \subset C \backslash\{0\}$ and $C$ is a closed convex cone, $C_{0}:=\overline{c o n e} H \subset C$. Using (2.5) with $y_{1}$ and $y_{2}$ replaced by $2 \gamma d\left(x, x^{\prime}\right) \sum_{i=1}^{n} h_{i}^{\prime}$ and $z_{1}-u_{n+1}$, we have

$$
\begin{aligned}
\zeta \cdot 2 \gamma d\left(x, x^{\prime}\right) \cdot n \kappa & \leq \zeta \cdot\left\|2 \gamma d\left(x, x^{\prime}\right) \sum_{i=1}^{n} h_{i}^{\prime}\right\| \\
& \leq\left\|z_{1}-u_{n+1}\right\| \\
& \leq\left(\left\|z_{1}\right\|+\eta\right), \quad \forall n \in \mathbb{N} .
\end{aligned}
$$

Since $\kappa, \zeta, \eta$ and $\gamma$ are positive constants it follows that $d\left(x, x^{\prime}\right)=0$ and so $x=x^{\prime}$.

Remark 3.1. Assumption (A3) in Proposition 3.2 can be relaxed to the condition that $F(x)$ is $C$-bounded for each $x \in \operatorname{dom} F$.

Lemma 3.3. Consider $\gamma>0$ and $F, H$ satisfying assumptions (A1)-(A4) with the associated constants $\kappa, \zeta, \eta>0$. Let $\left\{x_{n}\right\}_{n \in \mathbb{N}}$ be a decreasing sequence of dom $F$ with respect to $\preceq_{(F, \gamma, H)}$ ( $\preceq$ for short). Then $\left\{x_{n}\right\}_{n \in \mathbb{N}}$ converges to some $\bar{a} \in \operatorname{dom} F$, and

$$
F\left(x_{n}\right) \subset F(\bar{a})+C, \quad \forall n \in \mathbb{N} .
$$

The same assertion is also true for $\precsim_{(F, \gamma, H)}$ in place of $\preceq_{(F, \gamma, H)}$.

Proof. As the last assertion follows easily from the first, we only need to prove the results regarding $\preceq$. First, we show that $\left\{x_{n}\right\}_{n \in \mathbb{N}}$ is Cauchy. Suppose not, without loss of generality, we may assume that there exists $\varepsilon>0$ such that

$$
d\left(x_{n}, x_{n+1}\right)>\epsilon, \quad \forall n \in \mathbb{N} .
$$

Since $\left\{x_{n}\right\}_{n \in \mathbb{N}}$ is decreasing, there are sequences $\left\{y_{n}\right\}_{n \in \mathbb{N}}$, and $\left\{h_{n}\right\}_{n \in \mathbb{N}} \subset H$ such that

$$
\begin{aligned}
y_{n} \in F\left(x_{n}\right), & \forall n \in \mathbb{N} \\
\gamma d\left(x_{n}, x_{n+1}\right) h_{n} \leq_{C} y_{n}-y_{n+1}, & \forall n \in \mathbb{N} .
\end{aligned}
$$


By (3.19), there exists a sequence $\left\{u_{n}\right\}_{n \in \mathbb{N}} \subset Y$ such that

$$
\left\|u_{n}\right\| \leq \eta \text { and } u_{n} \leq_{C} y_{n} .
$$

It follows from (3.32) that

$$
\begin{aligned}
y_{1}-u_{n+1} & \geq_{C} y_{1}-y_{n+1}=\sum_{i=1}^{n}\left(y_{i}-y_{i+1}\right) \\
& \geq_{C} \sum_{i=1}^{n} \gamma d\left(x_{i}, x_{i+1}\right) h_{i} \\
& =\sum_{i=1}^{n} \gamma \alpha_{i} h_{i}=\gamma \beta_{n}\left(\sum_{i=1}^{n} \frac{\alpha_{i}}{\beta_{n}} h_{i}\right), \quad \forall n \in \mathbb{N},
\end{aligned}
$$

where $\alpha_{i}:=d\left(x_{i}, x_{i+1}\right)$ and $\beta_{n}:=\sum_{i=1}^{n} \alpha_{i}$. Noting by (3.30) that $\beta_{n}>n \epsilon$, and it follows from (2.5), (3.33) together with the assumed (A1) that for any $n \in \mathbb{N}$,

$$
\begin{aligned}
\left\|y_{1}\right\|+\eta & \geq\left\|y_{1}-u_{n+1}\right\| \\
& \geq \zeta \cdot \gamma \beta_{n}\left\|\sum_{i=1}^{n} \frac{\alpha_{i}}{\beta_{n}} h_{i}\right\| \geq \zeta \cdot \gamma n \epsilon\left\|\sum_{i=1}^{n} \frac{\alpha_{i}}{\beta_{n}} h_{i}\right\| \geq \zeta \cdot \gamma n \epsilon \cdot \kappa .
\end{aligned}
$$

But this is impossible because $\kappa, \zeta, \eta$ and $\gamma$ are positive constants while $n$ is arbitrary. Therefore $\left\{x_{n}\right\}_{n \in \mathbb{N}}$ is Cauchy and hence converges to some $\bar{a} \in M$.

Moreover, note that for any fixed $n \in \mathbb{N},\left\{x_{k}\right\}_{k \geq n}$ also converges to $\bar{a}$; this together with (3.2) and (3.6) implies that for any $y \in F\left(x_{n}\right),\left\{\left(x_{k}, y\right): k \geq n\right\}$ is a sequence in epi $F$ and converges to $(\bar{a}, y)$. Hence $(\bar{a}, y) \in$ epi $F$ thanks to the assumption that epi $F$ is closed. Therefore $\bar{x} \in \operatorname{dom} F$ and $y \in F(\bar{a})+C$ for each $y \in F\left(x_{n}\right)$, that is, (3.29) is true.

Next we present a set-valued version of Ekeland's variational principle type.

TheOREM 3.4. Suppose all the assumptions in Lemma 3.3 are satisfied. Then $\operatorname{dom} F$ has the domination property with respect to $\precsim_{(F, \gamma, H)}$, namely, for each $x_{0} \in$ $\operatorname{dom} F$, there is $\bar{x} \in \operatorname{dom} F$ such that

$$
\bar{x} \precsim_{(F, \gamma, H)} x_{0} \quad \text { and } \quad \bar{x} \in \operatorname{Min}\left(\operatorname{dom} F, \precsim_{(F, \gamma, H)}\right) ;
$$

in other words,

$$
F\left(x_{0}\right) \subset \cap_{\gamma^{\prime} \in(0, \gamma)}\left[F(\bar{x})+\gamma^{\prime} d\left(x_{0}, \bar{x}\right) H+C\right]
$$

and

$$
F(\bar{x}) \nsubseteq \cap_{\gamma^{\prime} \in(0, \gamma)}\left[F(x)+\gamma^{\prime} d(x, \bar{x}) H+C\right], \quad \forall x \in(\operatorname{dom} F) \backslash\{\bar{x}\}
$$

Proof. We use $\precsim$ to denote $\precsim(F, \gamma, H)$ for simplicity. By Proposition 3.2 , $\precsim$ is a partial order on $\operatorname{dom} F$. Let $\left\{x_{n}\right\}_{n \in \mathbb{N}}$ be a decreasing sequence of $\operatorname{dom} F$ with respect to $\preceq$. By Lemma 3.3, $\left\{x_{n}\right\}_{n \in \mathbb{N}}$ converges to some $\bar{a} \in \operatorname{dom} F$ and (3.29) holds. By Lemma 2.2, we need only to show that

$$
\bar{a} \precsim x_{n}, \quad \forall n \in \mathbb{N} .
$$


Let $n \in \mathbb{N}$ be fixed and consider any $\gamma^{\prime} \in(0, \gamma)$. Since $\left\{x_{k}\right\}_{k \geq n}$ converges to $\bar{a}$, there exists $k^{\prime} \geq n$ such that

$$
d\left(x_{k^{\prime}}, \bar{a}\right) \leq \frac{\gamma-\gamma^{\prime}}{\gamma+\gamma^{\prime}} d\left(x_{n}, \bar{a}\right) .
$$

Noting that $x_{k^{\prime}} \precsim x_{n}$ and $\frac{\gamma+\gamma^{\prime}}{2} \in(0, \gamma)$, it follows that for any $y \in F\left(x_{n}\right)$, there exist $y^{\prime} \in F\left(x_{k^{\prime}}\right)$ and $h^{\prime} \in H$ such that

$$
\frac{\gamma+\gamma^{\prime}}{2} d\left(x_{n}, x_{k^{\prime}}\right) h^{\prime} \leq_{C} y-y^{\prime} .
$$

By (3.29), there exists $\bar{y} \in F(\bar{a})$ such that

$$
\bar{y} \leq_{C} y^{\prime} .
$$

And by (3.40), we have

$$
d\left(x_{n}, x_{k^{\prime}}\right) \geq d\left(x_{n}, \bar{a}\right)-d\left(x_{k^{\prime}}, \bar{a}\right) \geq \frac{2 \gamma^{\prime}}{\gamma+\gamma^{\prime}} d\left(x_{n}, \bar{a}\right) .
$$

Hence, by (3.41), (3.42) and (3.43), we have

$$
\gamma^{\prime} d\left(x_{n}, \bar{a}\right) h^{\prime} \leq_{C} y-\bar{y}
$$

Thus $\bar{a} \precsim x_{n}$ by (3.3). So (3.39) holds and we complete the proof. $\square$

REMARK 3.2. Ekeland's variational principle (that we stated at the beginning) can be deduced immediately from Theorem 3.4 and Example 3.2 (with $n=1$ and $\left.f_{1}(x)=f(x)-\inf _{M} f(\cdot)\right)$ and the fact that

$$
\cap_{\gamma^{\prime} \in(0, \gamma)}\left[\left\{f_{1}(\bar{x})\right\}+\gamma^{\prime} d\left(x_{0}, \bar{x}\right)+[0,+\infty)\right]=\left\{f_{1}(\bar{x})\right\}+\gamma d\left(x_{0}, \bar{x}\right)+[0,+\infty)
$$

and

$$
\cap_{\gamma^{\prime} \in(0, \gamma)}\left[\left\{f_{1}(x)\right\}+\gamma d(x, \bar{x})+[0,+\infty)\right]=\left\{f_{1}(x)\right\}+\gamma d(x, \bar{x})+[0,+\infty) .
$$

Next we discuss the domination property of $\operatorname{dom} F$ with respect to $\preceq_{(F, \gamma, H)}$. The following theorem is similar to (but distinct from) Theorem 3.4. In fact Example 3.1 has shown that $\preceq_{(F, \gamma, H)}$ and $\precsim_{(F, \gamma, H)}$ are distinct, even when dom $F$ has the domination property with respect to each of the two relations. Also there are examples to show that Theorem 3.5 would not longer be valid if (i), (ii) and (iii) are dropped.

Recall that the positive polar of $C$ is defined as

$$
C^{+}:=\left\{y^{*} \in Y^{*}:\left\langle y^{*}, y\right\rangle \geq 0, \forall z \in C\right\} .
$$

THEOREM 3.5. Suppose all the assumptions in Lemma 3.3 are satisfied, and that (at least) one of the following assertions holds:

(i) There exist $y_{0}^{*} \in S\left(C^{+}\right)$and $\xi \in(0,1)$ such that

$$
H \subset\left\{y \in Y: \xi\|y\| \leq\left\langle y_{0}^{*}, y\right\rangle\right\} .
$$

(ii) $H$ is bounded. 
(iii) $Y$ is reflexive.

Then dom $F$ has the domination property with respect to $\preceq_{(F, \gamma, H)}$, namely, for each $x_{0} \in \operatorname{dom} F$, there is $\bar{x} \in \operatorname{dom} F$ such that

$$
\bar{x} \preceq_{(F, \gamma, H)} x_{0} \quad \text { and } \quad \bar{x} \in \operatorname{Min}\left(\operatorname{dom} F, \preceq_{(F, \gamma, H)}\right) ;
$$

in other words,

$$
F\left(x_{0}\right) \subset F(\bar{x})+\gamma d\left(x_{0}, \bar{x}\right) H+C,
$$

and

$$
F(\bar{x}) \nsubseteq F(x)+\gamma d(x, \bar{x}) H+C, \quad \forall x \in(\operatorname{dom} F) \backslash\{\bar{x}\}
$$

Proof. We use $\preceq$ to denote $\preceq_{(F, \gamma, H)}$ for simplicity. By Proposition 3.2 , $\preceq$ is a partial order on $\operatorname{dom} F$. Let $\left\{x_{n}\right\}_{n \in \mathbb{N}}$ be a decreasing sequence of $\operatorname{dom} F$ with respect to $\preceq$. By Lemma 3.3, $\left\{x_{n}\right\}_{n \in \mathbb{N}}$ converges to some $\bar{a} \in \operatorname{dom} F$ and (3.29) holds. Similar to Theorem 3.4, we need only to show that

$$
\bar{a} \preceq x_{n}, \quad \forall n \in \mathbb{N} .
$$

Let $n_{0} \in \mathbb{N}$ be fixed. Since $\left\{x_{n}\right\}_{n \in \mathbb{N}}$ converges to $\bar{a}$, there exists a subsequence $\left\{z_{n}\right\}_{n \in \mathbb{N}} \subset\left\{x_{n}\right\}_{n \in \mathbb{N}}$ such that

$$
z_{1}=x_{n_{0}}
$$

and

$$
z_{n+1} \preceq z_{n} \text { and } d\left(z_{n+1}, \bar{a}\right)<\frac{1}{n+1}, \quad \forall n \in \mathbb{N} .
$$

For any fixed $y_{1} \in F\left(z_{1}\right)$, let $\left\{y_{n}\right\}_{n \in \mathbb{N}} \subset Y,\left\{h_{n}^{\prime}\right\}_{n \in \mathbb{N}} \subset H$ and $\left\{u_{n}\right\}_{n \in \mathbb{N}} \subset Y$ such that

$$
\begin{aligned}
y_{n} \in F\left(z_{n}\right), & \forall n \in \mathbb{N}, \\
\gamma d\left(z_{n}, z_{n+1}\right) h_{n}^{\prime} \leq_{C} y_{n}-y_{n+1}, & \forall n \in \mathbb{N}, \\
\left\|u_{n}\right\| \leq \eta \text { and } u_{n} \leq_{C} y_{n}, & \forall n \in \mathbb{N} .
\end{aligned}
$$

It follows that

$$
\begin{aligned}
y_{1}-u_{n+1} & \geq_{C} y_{1}-y_{n+1} \\
& \geq_{C} \sum_{i=1}^{n}\left[\gamma d\left(z_{i}, z_{i+1}\right) h_{i}^{\prime}\right]=\gamma \beta_{n}\left[\sum_{i=1}^{n} \frac{\alpha_{i}}{\beta_{n}} h_{i}^{\prime}\right]=\gamma \beta_{n} h_{n}^{\prime \prime}, \quad \forall n \in \mathbb{N},
\end{aligned}
$$

where $\alpha_{i}:=d\left(z_{i}, z_{i+1}\right), \beta_{n}:=\sum_{i=1}^{n} \alpha_{i}$ and $h_{n}^{\prime \prime}:=\sum_{i=1}^{n} \frac{\alpha_{i}}{\beta_{n}} h_{i}^{\prime} \in H$. Using (2.5) together with the properties of $\eta, \zeta$ and $\kappa$ given in (A1)-(A4), we get

$$
\left\|y_{1}\right\|+\eta \geq \zeta \cdot \gamma \beta_{n}\left\|h_{n}^{\prime \prime}\right\| \geq \zeta \cdot \gamma \beta_{n} \cdot \kappa, \quad \forall n \in \mathbb{N} .
$$

This implies that $\left\{\beta_{n}\right\}_{n \in \mathbb{N}}$ is bounded and hence converges to a finite limit, say $d_{0}$, that is, $d_{0}=\sum_{j=1}^{+\infty} d\left(z_{j}, z_{j+1}\right)<+\infty$. Since $\beta_{n}=\sum_{j=1}^{n} d\left(z_{j}, z_{j+1}\right) \geq d\left(z_{1}, \bar{a}\right)-$ $d\left(z_{n+1}, \bar{a}\right)>d\left(z_{1}, \bar{a}\right)-\frac{1}{n+1}$ for all $n \in \mathbb{N}$, it follow from (3.57) that, for each $n$,

$$
y_{1}-\gamma\left[d\left(z_{1}, \bar{a}\right)-\frac{1}{n+1}\right] h_{n}^{\prime \prime} \geq_{C} y_{1}-\gamma \beta_{n} h_{n}^{\prime \prime} \geq_{C} y_{n+1},
$$


that is,

$$
y_{1}-\gamma\left[d\left(z_{1}, \bar{a}\right)-\frac{1}{n+1}\right] h_{n}^{\prime \prime} \in y_{n+1}+C, \quad \forall n \in \mathbb{N} .
$$

Since $y_{n+1} \in F\left(z_{n+1}\right) \subset F(\bar{a})+C$ (by (3.54) and (3.29) applied to $z_{n+1}$ in place of $\left.x_{n}\right)$, it follows (3.60) that

$$
y_{1}-\gamma\left[d\left(z_{1}, \bar{a}\right)-\frac{1}{n+1}\right] h_{n}^{\prime \prime} \in F(\bar{a})+C
$$

and so

$$
\left(\bar{a}, y_{1}-\gamma\left[d\left(z_{1}, \bar{a}\right)-\frac{1}{n+1}\right] h_{n}^{\prime \prime}\right) \in \text { epi } F, \quad \forall n \in \mathbb{N} .
$$

We now split the proof into the following three cases.

(i) There exists $y_{0}^{*} \in S\left(C^{+}\right)$and $\xi \in(0,1)$ such that $(3.47)$ holds. It follows from (3.56), (3.57) and (3.47) that

$$
\left\|y_{1}\right\|+\eta \geq\left\langle y_{0}^{*}, y_{1}-u_{n+1}\right\rangle \geq\left\langle y_{0}^{*}, \gamma \sum_{i=1}^{n} \alpha_{i} h_{i}^{\prime}\right\rangle \geq \gamma \xi \sum_{i=1}^{n} \alpha_{i}\left\|h_{i}^{\prime}\right\|,
$$

for all $n$. This implies that $\sum_{i=1}^{+\infty} \alpha_{i}\left\|h_{i}^{\prime}\right\|$ is a convergent series. Moreover, for all $m, n \in \mathbb{N}$ with $m<n$, we have

$$
h_{n}^{\prime \prime}-h_{m}^{\prime \prime}=\sum_{i=1}^{m} \alpha_{i} h_{i}^{\prime}\left(\frac{1}{\beta_{n}}-\frac{1}{\beta_{m}}\right)+\sum_{i=m+1}^{n} \frac{\alpha_{i} h_{i}^{\prime}}{\beta_{n}} .
$$

Hence

$$
\begin{aligned}
\left\|h_{n}^{\prime \prime}-h_{m}^{\prime \prime}\right\| & \leq \frac{\beta_{n}-\beta_{m}}{\beta_{n} \cdot \beta_{m}}\left(\sum_{i=1}^{m} \alpha_{i}\left\|h_{i}^{\prime}\right\|\right)+\frac{1}{\beta_{n}}\left(\sum_{i=m+1}^{n} \alpha_{i}\left\|h_{i}^{\prime}\right\|\right) \\
& \leq \frac{\beta_{n}-\beta_{m}}{\beta_{n} \cdot \beta_{m}}(\gamma \xi)^{-1}\left(\left\|y_{1}\right\|+\eta\right)+\frac{1}{\beta_{n}}\left(\sum_{i=m+1}^{n} \alpha_{i}\left\|h_{i}^{\prime}\right\|\right) .
\end{aligned}
$$

Since $\beta_{m}, \beta_{n}$ converge to $d_{0}$ and $\sum_{i=m+1}^{n} \alpha_{i}\left\|h_{i}^{\prime}\right\|$ converges to 0 when $m \rightarrow \infty$, the sequence $\left\{h_{n}^{\prime \prime}\right\}$ is Cauchy. Let $h_{0}:=\lim _{n \rightarrow \infty} h_{n}^{\prime \prime}$; then $h_{0} \in H$, by (3.62) we get

$$
\left(\bar{a}, y_{1}-\gamma d\left(z_{1}, \bar{a}\right) h_{0}\right) \in \text { epi } F .
$$

Since $y_{1}$ is arbitrary in $F\left(z_{1}\right)$ and $z_{1}=x$, this implies that (3.51) holds (see $(3.5))$.

(ii) $H$ is bounded, namely $\sup _{H}\|\cdot\|<+\infty$. For any $v \in H+C$, there exist $v_{1} \in H$ such that $v_{1} \leq_{C} v$. Then, by (2.5), we have

$$
\|v\| \geq\left\|v_{1}\right\| \cdot \zeta \geq \kappa \cdot \zeta,
$$

and so

$$
\inf _{H+C}\|\cdot\| \geq \kappa \cdot \zeta>0 .
$$


This implies that $c l(H+C)$ (which is a closed convex set) does not contain 0 . By the separation theorem, there exists $y_{0}^{*} \in Y^{*}$ with $\left\|y_{0}^{*}\right\|=1$ such that

$$
\inf _{\operatorname{cl}(H+C)}\left\langle y_{0}^{*}, \cdot\right\rangle>0 .
$$

Thus $y_{0}^{*} \in S\left(C^{+}\right)$and

$$
\inf _{H}\left\langle y_{0}^{*}, \cdot\right\rangle>0
$$

Hence one can pick $\xi \in(0,1)$ such that

$$
\xi<\frac{\inf _{H}\left\langle y_{0}^{*}, \cdot\right\rangle}{\sup _{H}\|\cdot\|} .
$$

Now it is easy to verify (3.47). This shows that (ii) is a special case of (i).

(iii) $Y$ is reflexive. By (3.59) and (3.56) we note that, for all $n$,

$$
\gamma\left[d\left(z_{1}, \bar{a}\right)-\frac{1}{n+1}\right] h_{n}^{\prime \prime} \leq_{C} y_{1}-y_{n+1} \leq_{C} y_{1}-u_{n+1} .
$$

Using (2.5), we have

$$
\zeta \cdot \gamma\left[d\left(z_{1}, \bar{a}\right)-\frac{1}{n+1}\right]\left\|h_{n}^{\prime \prime}\right\| \leq\left\|y_{1}-u_{n+1}\right\| \leq\left\|y_{1}\right\|+\eta .
$$

Thus $\left\{h_{n}^{\prime \prime}\right\}$ is bounded and so has a weak-cluster point $\bar{h}_{0}$ in the closed convex set $H$ (thanks to the reflexivity assumption) We claim that

$$
\left(\bar{a}, y_{1}-\gamma d\left(z_{1}, \bar{a}\right) \bar{h}_{0}\right) \in \operatorname{epi} F
$$

(that is, (3.65) holds with $h_{0}$ replaced by $\bar{h}_{0}$ and so one completes the proof as that in (i)). To establish the claim, we take any $n_{1} \in \mathbb{N}$ and let $H_{1}:=\operatorname{co}\left(\left\{h_{n}^{\prime \prime}\right\}_{n>n_{1}}\right)$. Then $\operatorname{cl}\left(H_{1}\right)$ is a weak closed convex set and hence $\bar{h}_{0}=w^{*}-\lim _{n \rightarrow+\infty} h_{n}^{\prime \prime} \in \operatorname{cl}\left(H_{1}\right)$. Therefore there exist $n_{2}>n_{1}$ and $\lambda_{n_{1}+1}, \cdots, \lambda_{n_{2}} \geq 0$ such that $\sum_{i=n_{1}+1}^{n_{2}} \lambda_{i}=1$ and

$$
\left\|\bar{h}_{0}-\bar{h}_{1}\right\|<1,
$$

where $\bar{h}_{1}:=\sum_{i=n_{1}+1}^{n_{2}} \lambda_{i} h_{i}^{\prime \prime} \in H_{1} \subset H$. By (3.60) we note that for any $n \in \overline{n_{1}+1, n_{2}}$,

$$
\begin{aligned}
y_{1}-\gamma\left[d\left(z_{1}, \bar{a}\right)-\frac{1}{n_{1}+1}\right] h_{n}^{\prime \prime} & \geq_{C} y_{1}-\gamma\left[d\left(z_{1}, \bar{a}\right)-\frac{1}{n+1}\right] h_{n}^{\prime \prime} \\
& \geq_{C} y_{n+1} \geq_{C} y_{n_{2}+1}
\end{aligned}
$$

and hence

$$
y_{1}-\gamma\left[d\left(z_{1}, \bar{a}\right)-\frac{1}{n_{1}+1}\right] \bar{h}_{1} \in y_{n_{2}+1}+C \subset F\left(z_{n_{2}+1}\right)+C .
$$

Inductively, we construct sequences $\left\{n_{k}\right\}_{k \in \mathbb{N}}$ and $\left\{\bar{h}_{k}\right\}_{k \in \mathbb{N}}$ such that

$$
\begin{aligned}
1 & \leq n_{1}<n_{2}<\cdots, \\
\left\|\bar{h}_{0}-\bar{h}_{k}\right\| & <\frac{1}{k}, \quad \forall k \in \mathbb{N},
\end{aligned}
$$


and

$$
y_{1}-\gamma\left[d\left(z_{1}, \bar{a}\right)-\frac{1}{n_{k}+1}\right] \bar{h}_{k} \in F\left(z_{n_{(k+1)}+1}\right)+C, \quad \forall k \in \mathbb{N} .
$$

Therefore $\left(z_{n_{(k+1)}+1}, y_{1}-\gamma\left[d\left(z_{1}, \bar{a}\right)-\frac{1}{n_{k}+1}\right] \bar{h}_{k}\right) \in e p i F$ for all $k \in \mathbb{N}$ and $\left\{\left(z_{n_{(k+1)}+1}, y_{1}-\gamma\left[d\left(z_{1}, \bar{a}\right)-\frac{1}{n_{k}+1}\right] \bar{h}_{k}\right)\right\}_{k \in \mathbb{N}}$ converges to $\left(\bar{a}, y_{1}-\gamma d\left(z_{1}, \bar{a}\right) \bar{h}_{0}\right)$. Thus (3.72) holds since epi $F$ is closed.

REMARK 3.3.

(a) The idea of using partial order in the Ekeland's Variational Principle related results has been used by many authors including Ekeland [14] himself, Bishop-Phelps [6], Brondsted-Rockafellar [8], Dancs-Hegedus-Medvegyev [11], Turinici [35], and Hamel-Tammer [18].

(b) Most of the extensions (see [3, Theorem 1],[4, Theorem 3.4], [9, Chpater 4] [16, Theorem 3.1 \& 3.2], [18, Theorem $4.1 \&$ 4.2], [38, Theorem 4.1 \& 4.2]) and references therein) of the Ekeland principle take the following form: Under some suitable conditions, for any $\gamma>0, \xi \in C \backslash\{0\}$, $\left(x_{0}, y_{0}\right) \in \operatorname{graph} F \subset M \times Y$, there exists $(\bar{x}, \bar{y}) \in \operatorname{graph} F$ such that

$$
\begin{aligned}
& y_{0} \in \bar{y}+\gamma d\left(x_{0}, \bar{x}\right) \xi+C, \quad \bar{y} \in \operatorname{Min}\left(F(\bar{x}), \leq_{C}\right), \\
& \bar{y} \notin y+\gamma d(\bar{x}, x) \xi+C, \quad \forall(x, y) \in \operatorname{graph} F,(x, y) \neq(\bar{x}, \bar{y}) .
\end{aligned}
$$

A novelty of our approach here is to replace the singleton $\{\xi\}$ but allowing $\xi$ in (3.79) to be selected from a set $H$ satisfying (A1) and (A2). Thus, instead of approaches of earlier authors asserting relations between elements of the values of the set-valued function F, our extension (reported in Theorem 3.4 and Theorem 3.5) of the Ekeland principle are directly expressed in terms of values (as entities) of $F$. The idea of replacing a singleton by a set or a suitable set-valued function has very recently been used by Bednarczuk-Zagrodny [5] and Gutiérrez-Jiménez-Novo [15] in their extended Ekeland's variational principles for vector-valued (single-valued) functions. Our discussion on the issue of error bounds in the next section will further shed light on using a set $H$ instead of a singleton in our extensions of the Ekeland's variational principle.

The notion of considering relations between values of $F$ has also been used by Kuroiwa [21, 22] when he studied set-valued optimization problems and their dual problems.

(c) The assumption (A2) plays an important role in Theorem 3.5 especially for (3.51) and also it ensures that $\preceq_{(F, \gamma, H)}$ and $\precsim_{(F, \gamma, H)}$ defined in Definition 3.1 are anti-symmetric.

4. Error Bounds of Systems. In this section we consider a Banach space $X$ and study the inequality system (1.3) defined by proper lsc functions from $X$ to $(-\infty,+\infty]$. To avoid the triviality, we always assume that

$$
D:=\cap_{i=1}^{n} \operatorname{dom} f_{i} \neq \emptyset \text {. }
$$

Let $S$ be the solution set (defined in (1.5)). For each $x \in X$, Let $I_{>}(x)$ denote the set of "infeasibility indices" for $x$ while $I_{\geq}(x)$ denotes that of "boundary indices"; they 
are respectively defined by

$$
\begin{aligned}
& I_{>}(x):=\left\{i \in \overline{1, n}: f_{i}(x)>0\right\}, \\
& I_{\geq}(x):=\left\{i \in \overline{1, n}: f_{i}(x) \geq 0\right\} .
\end{aligned}
$$

Let $\gamma>0$, and we say that $u \in X \backslash S$ has the $\gamma$-descent property if there exists $\hat{u} \in X \backslash\{u\}$ satisfying the following properties

$$
\begin{aligned}
& I_{>}(\hat{u}) \subset I_{>}(u), \\
& f_{i}(\hat{u}) \leq f_{i}(u), \quad \forall i \in I_{>}(u), \\
& \gamma d(u, \hat{u}) \leq \sum_{i \in I_{>}(u)}\left(f_{i}(u)_{+}-f_{i}(\hat{u})_{+}\right) .
\end{aligned}
$$

By (4.3) and (4.4) it is clear that

$$
f_{i}(\hat{u})_{+} \leq f_{i}(u)_{+}, \quad \forall i \in \overline{1, n}
$$

(both sides are zero if $i \notin I_{>}(u)$ ). This together with (4.5) implies that

$$
\gamma d(u, \hat{u}) \leq \sum_{i=1}^{n}\left(f_{i}(u)_{+}-f_{i}(\hat{u})_{+}\right) .
$$

EXAmple 4.1. Let $f$ be be a proper function from $X$ to $(-\infty,+\infty]$. For $x \in X$ with $f(x) \in \mathbb{R}$ and $v \in X$, recall that (cf. [28]) upper Dini-directional derivative of $f$ at $x$ in direction $v$ is defined by

$$
\bar{d}^{+} f(x)(v):=\limsup _{t \rightarrow 0^{+}} \frac{f(x+t v)-f(x)}{t} .
$$

Let $\gamma>0$ and $u \in X \backslash S$. If there exists $v \in X$ with $\|v\|=1$ such that

$$
\begin{aligned}
& \bar{d}^{+} f_{i}(u)(v) \text { exists and finite for each } i \in \overline{1, n}, \\
& \bar{d}^{+} f_{i}(u)(v)<0, \quad \forall i \in I_{\geq}(u), \\
& \sum_{i \in I_{>}(u)} \bar{d}^{+} f_{i}(u)(v) \leq-\gamma .
\end{aligned}
$$

Then $u$ has the $\gamma^{\prime}$-descent property for any $\gamma^{\prime} \in(0, \gamma)$. To see this, let $\gamma^{\prime} \in(0, \gamma)$ be fixed. By (4.11) and (4.10) (applied to the indices $i$ in $I_{>}(u)$ ), there exists a series of positive numbers $\left\{\gamma_{i}^{\prime}: i \in I_{>}(u)\right\}$ such that

$$
\bar{d}^{+} f_{i}(u)(v)<-\gamma_{i}^{\prime}, \quad \forall i \in I_{>}(u),
$$

and

$$
\sum_{i \in I_{>}(x)} \gamma_{i}^{\prime}=\gamma^{\prime}
$$

For each $i \in \overline{1, n}$, we select $t_{i}>0$ in the following way:

(a) If $f_{i}(u)>0$, then by (4.12), there exists $t_{i}>0$ such that

$$
0<f_{i}(u+t v)<f_{i}(u)-\gamma_{i}^{\prime} t, \quad \forall t \in\left(0, t_{i}\right] .
$$


(b) If $f_{i}(u)=0$ then, by (4.10), there exists $t_{i}>0$ such that

$$
f_{i}(u+t v)<0, \quad \forall t \in\left(0, t_{i}\right] .
$$

(c) If $f_{i}(u)<0$ then, by (4.9), there exists $t_{i}>0$ such that

$$
f_{i}(u+t v)<0, \quad \forall t \in\left(0, t_{i}\right] .
$$

Having specified $t_{i}>0$ for all $i \in \overline{1, n}$, let $\hat{t}:=\min \left\{t_{i}: i \in \overline{1, n}\right\}$ and $\hat{u}:=u+\hat{t} v$. We note that the following equivalence holds for all $i$ :

$$
f_{i}(\hat{u})>0 \Longleftrightarrow f_{i}(u)>0,
$$

that is $I_{>}(\hat{u})=I_{>}(u)((4.3)$ holds). Further, by (4.13) and (4.14), we have (4.4) and

$$
\gamma^{\prime} d(u, \hat{u}) \leq \sum_{i \in I_{>}(u)}\left(f_{i}(u)_{+}-f_{i}(\hat{u})_{+}\right) .
$$

Therefore $u$ has the $\gamma^{\prime}$-descent property.

The following result was established (based on a result of Hamel [17, Theorem $2($ ii)] $)$ in [28] for the special case when $n=1$ and $W=\emptyset$.

THeOREM 4.1. Let $X, f_{1}, f_{2}, \cdots, f_{n}, D$ and $S$ be as at the beginning of this section. Suppose that there exist positive constants $\tau_{1}, \gamma$ and a subset $W$ of $X$ satisfying the following two conditions:

(i)

$$
d(w, S) \leq \tau_{1} \sum_{i=1}^{n} f_{i}(w)_{+}, \quad \forall w \in W .
$$

(ii) Each $u \in X \backslash(W \cup S)$ has the $\gamma$-descend property (with the corresponding $\hat{u} \neq u)$.

Then the inequality system (1.3) has an error bound $\tau:=\max \left\{\gamma^{-1}, \tau_{1}\right\}$.

Proof. Let $x_{0}$ be an arbitrary element of $X$. We have to show that the following inequality

$$
d\left(x_{0}, S\right) \leq \tau \sum_{i=1}^{n} f_{i}\left(x_{0}\right)_{+}
$$

We suppose without loss of generality that $x_{0} \in D \backslash(W \cup S)$. We shall apply Theorem 3.5 with the following data: $Y=\mathbb{R}^{n}$ with the partial order defined by $C:=\mathbb{R}_{+}^{n}$ and the $l_{1}$-norm. Clearly, if $y \in \mathbb{R}_{+}^{n} \backslash\{0\}$ and $\lambda \geq 0$ then

$$
\lambda \leq\|y\|_{1} \Longleftrightarrow \lambda \frac{y}{\|y\|_{1}} \leq_{\mathbb{R}_{+}^{n}} y .
$$

Let $H:=\mathbb{R}_{+}^{n} \cap\left\{y:\|y\|_{1}=1\right\}$. Thus

$$
d(0, H)=1, \quad \overline{\text { cone }} H=\mathbb{R}_{+}^{n} \quad \text { and } \quad \measuredangle\left(\overline{\text { cone } H},-\mathbb{R}_{+}^{n}\right)=1 .
$$

Let $F: D \rightarrow Y$ be defined by $F(x)=\left(f_{1}(x)_{+}, f_{2}(x)_{+}, \cdots, f_{n}(x)_{+}\right)$for $x \in D$. Thus epi $F$ is closed (thanks to the assumption that each $f_{i}$ is lsc). Together with (4.21), we see that (A1)-(A4) are satisfied by the data. Hence Proposition 3.2 and Theorem 
3.5 are applicable with $\preceq:=\preceq_{(F, \gamma, H)}$. In particular, there exists $u \in D$ satisfying $u \preceq x_{0}$ and $z \npreceq u$ for all $z \in D \backslash\{u\}$ (that is $u$ is a minimum element of $D$ ). Thus, by definitions, there exists $h_{1} \in H$ such that

$$
\gamma d\left(x_{0}, u\right) h_{1} \leq_{\mathbb{R}_{+}^{n}} F\left(x_{0}\right)-F(u),
$$

and so

$$
\gamma d\left(x_{0}, u\right)=\left\|\gamma d\left(x_{0}, u\right) h_{1}\right\|_{1} \leq\left\|F\left(x_{0}\right)-F(u)\right\|_{1} .
$$

We claim that $u \in W \cup S$. If not, then, by assumption (ii), $u$ has the $\gamma$-descent property with the corresponding $\hat{u} \neq u$ : (4.3)-(4.7) hold. Clearly, (4.7) can be rewritten as $\gamma d(u, \hat{u}) \leq\|F(u)-F(\hat{u})\|_{1}$, and it follows from (4.20) that $\gamma d(u, \hat{u}) h_{2} \leq F(u)-F(\hat{u})$, where $h_{2}:=\frac{F(u)-F(\hat{u})}{\|F(u)-F(\hat{u})\|_{1}}$. This implies that $\hat{u} \preceq u$, contradicting the minimality of $u$. This shows that $u \in W \cup S$. If $u \in S$ then $F(u)=0$ and (4.23) entails that

$$
\gamma d\left(x_{0}, S\right) \leq\left\|F\left(x_{0}\right)\right\|_{1}=\sum_{i=1}^{n} f_{i}\left(x_{0}\right)_{+} .
$$

and so (4.19) holds in this case. It remains to consider the case when $u \in W$. Then, by (4.18), one has $\tau_{1}^{-1} d(u, S) \leq\|F(u)\|_{1}$ and it follows from (4.20) that, for $h_{3}=\frac{F(u)}{\|F(u)\|_{1}}$,

$$
\tau_{1}^{-1} d(u, S) h_{3} \leq_{\mathbb{R}_{+}^{n}} F(u)
$$

This and (4.22) imply that

$$
\min \left\{\gamma, \tau_{1}^{-1}\right\}\left[d\left(x_{0}, u\right) h_{1}+d(u, S) h_{3}\right] \leq_{\mathbb{R}_{+}^{n}} F\left(x_{0}\right) .
$$

and so $\min \left\{\gamma, \tau_{1}^{-1}\right\}\left[d\left(x_{0}, u\right)+d(u, S)\right] \leq\left\|F\left(x_{0}\right)\right\|_{1}$. Thus (4.19) is seen to be true. $\mathrm{Q}$

Acknowledgements. The authors would like to thank Prof. C. C. Chou for his stimulating discussion, and thank referees for their constructive comments leading to many improvements and for pointing out the references [3, 5, 15, 16, 21, 22, 38].

\section{REFERENCES}

[1] D. Aze, A survey on error bounds for lower semicontinuous functions, Proceedings of 2003 MODE-SMAI Conference, ESAIM Proc., 13, pp. 1-17, EDP Sci., Les Ulis, 2003.

[2] D. Aze, J.N. Corvellec, On the sensitivity analysis of Hoffman constants for systems of linear inequalities, SIAM J. Optim. 12 (2002), pp. 913-927.

[3] T. Q. Bao, B. S. Mordukhovich, Variational principle for set-valued mappings with applications to multiobjective optimization, Control and Cybernetics, 36(2007), pp. 531-562.

[4] T. Q. Bao, B. S. Mordukhovich, Relative Pareto minimizers for multiobjective problems: existence and optimality conditions, Math. Program., Ser. A 122 (2010), pp. 301-347.

[5] E. Bednarczuk, D. Zagrodny, Vector variational principle, Archiv der Mathematik, 93(2009), pp. 577-586.

[6] E. Bishop, R.R. Phelps, The support functionals of a convex set, Proc. Sympos. Pure Math., 7 (1963), pp. 27-35.

[7] P. Bosch, A. Jourani, R. Henrion, Sufficient conditions for error bounds and applications, Appl. Math. Optim. 50 (2004), pp. 161-181.

[8] A. Brøndsted, R. T. Rockafellar, On the subdifferentiability of convex functions, Proc. Amer. Math. Soc., 16 (1965), pp. 605-611.

[9] G. Y. Chen, X. X. Huang, X. Q. Yang, Vector Optimization, Lecture Notes in Economic and Mathematical Systems, 541, Springer-Verlag, Berlin, 2005.

[10] J. N. Corvellec, V. V. Motreanu, Nonlinear error bounds for lower semicontinuous functions on metric spaces, Math. Program., Ser. A 114 (2008), pp. 291-319. 
[11] S. Dancs, M. Hegedus, and P. Medvegyev, A general ordering and fixed point principle in complete metric spaces, Acta Sci. Math. 46 (1983), pp. 381-388.

[12] S. Deng, Global error bounds for convex inequality systems in Banach spaces, SIAM J. Control Optim. 36 (1998), pp. 1240-1249.

[13] I. Ekeland, Remarques sur les problems variationels 1, CRAS, 275 (1972), pp. 1057-1059.

[14] I. Ekeland, On the variational principle, J. Math. Anal. Appl., 47 (1974), pp. 324-353.

[15] C. Gutiérrez, B. Jiménez, V. Novo, A set-valued Ekeland's variational principle in vector optimization, SIAM J. Control Optim., 47 (2008), pp. 883-903.

[16] T. X. D. Ha, Variants of the Ekeland variational principle for a set-valued map involving the Clarke normal cone, J. Math. Anal. Appl., 316 (2006), pp. 346-356.

[17] A. Hamel, Remarks to equivalent formulation of Ekelands variational principle, Optimization, 31 (1994), pp. 233-238.

[18] A. H. Hamel, C. Tammer, Minimal elements for product orders, Optimization, 57 (2008), pp. 263-275.

[19] A. J. Hoffman, On approximate solutions of systems of linear inequalities, J. Research Nat. Bur. Standards 49 (1952), pp. 263-265.

[20] J. L. Kelley, General Topology, Springer-Verlag, New York, 1955.

[21] D. Kuroiwa, On set-valued optimization, Nonlinear Analysis, 47(2001), pp. 1395-1400.

[22] D. Kuroiwa, Existence theorems of set optimization with set-valued maps, J. Inf. Optim. Sci., 24(2003), pp. 73-84.

[23] A. Lewis, J. S. Pang, Error bounds for convex inequality systems, in Generalized Convexity, Generalized Monotonicity: Recent Results, Proceedings of the Fifth Symposium on GeneralizedCon vexity, Luminy, June 1996, J.-P. Crouzeix, J.-E. Martinez-Legaz, and M. Volle, eds., Kluwer Academic Publishers, Dordrecht, 1997, pp. 75-100.

[24] C. G. Liu, K. F. Ng, W. H. Yang, Merit functions in vector optimization, Math. Program. Ser. A, 119 (2009), pp. 215-237.

[25] D. T. Luc, Theory of Vector Optimization, Lecture Notes in Economics and Mathematical Systems 319, Springer, Berlin, Germany, 1989.

[26] R. E. Megginson, An Introduction to Banach Space Theory, Springer, New York, 1998.

[27] K. F. Ng, W. H. Yang, Regularities and their relations to error bounds, Math. Program. Ser. A, 99 (2004), pp. 521-538.

[28] K. F. Ng, X. Y. Zheng, Error bounds for lower semicontinuous functions in normed spaces, SIAM Journal on Optimization, 12 (2001), pp. 1-17.

[29] H. V. Ngai, M. Théra, Error bounds in metric spaces and application to the perturbation stability of metric regularity, SIAM J. Optim., 19 (2008), pp. 1-20.

[30] H. V. Ngai, M. Théra, Error bounds for systems of lower semicontinuous functions in Asplund spaces Math. Program., Ser. B, 116 (2009), pp. 397-427.

[31] J. S. Pang, Error bounds in mathematical programming, Math. Program. Ser. B, 79 (1997), pp. 299-332.

[32] A. L. Peressini, Ordered topological vector spaces, Harper \& Row, New York-London, 1967.

[33] R. R. Phelps, Convex Functions, Monotone Operaters and Differentiability, Springer, Berlin, 1989.

[34] H. H. Schaefer,Banach Lattices and Positive Operators, Springer-Verlag, New York-Heidelberg, 1974.

[35] M. Turinici, Maximality principles and mean-value theorems, Anais Acad. Brasil. Ciencias 53 (1981), pp. 653-655.

[36] Z. Wu, J. J. Ye, Sufficient conditions for error bounds, SIAM J. Optim. 12 (2001/02), pp. 421435.

[37] C. Zălinescu, Convex Analysis in General Vector Spaces, World Scientific, Singapore, 2002.

[38] J. Zhu, C. K. Zhong, Y. J. Cho, Generalized variational principle and vector optimization, JOTA, 106(2000), pp. 201-217. 